\title{
PRE-OPERATIVE IDENTIFICATION OF SLEEP APNEA RISK IN ELECTIVE SURGICAL PATIENTS
}

Hongbo Yuan Ph.D.*, Barnaby Ward F.R.C.A.*, Frances Chung F.R.C.P.C.*, Joyce Ho*, Leonid Kayumov Ph.D. $\dagger$ and Colin Shapiro F.R.C.P.C $\dagger$

Department of Anesthesiology*, Department of Psychiatry†, Toronto Western Hospital, University Health Network, 399 Bathurst Street, Toronto, Ontario, Canada. M5T 2S8

INTRODUCTION: Sleep apnea is a disorder associated with increased risk and morbidity during the perioperative period. The purpose of this study is to use the Berlin questionnaire to estimate the prevalence of sleep apnea in patients undergoing elective surgery.

METHODS: After Institutional Ethics Board approval, patients were asked to complete the Berlin questionnaire as a part of their preoperative assessment. Inclusion criteria a) patients with ASA I - IV; b) patients scheduled for elective procedures in general surgery, orthopedics, urology, plastic surgery, ophthalmology or neurosurgery. Exclusion criteria: patients undergoing surgery under neuroleptic or local anesthesia. Physical data regarding blood pressure and body mass index was also recorded. Patients were stratified into high or low risk groups for sleep apnea on the basis of their responses and physical data. The high and low risk groups for sleep apnea as identified by the Berlin questionnaire were compared. All patients deemed at high risk of sleep apnea by the Berlin questionnaire were offered an appointment for overnight polysomnographic testing for diagnosis of sleep apnea. Demographic data was analysed using the Chi square test and Student $t$ test.

RESULTS: Of 318 screened patients, the Berlin questionnaire identified 76 patients (24\%, 95\% CI 19- 29\%) at high risk of sleep apnea (see Table). Ten patients were known to have sleep apnea. The Berlin questionnaire correctly identified all these patients as being at high risk. There was a significantly higher incidence of uncontrolled pain in the high-risk group $(P=0.01)$ compared with the low-risk group. Analysis for perioperative adverse events did not show any significant cardiac, respiratory or neurological morbidity in the high-risk group compared to the low-risk group.

DISCUSSION: More than one in five elective patients were found to be at high risk of sleep apnea. The Berlin questionnaire correctly identified all patients previously diagnosed with sleep apnea as being at high risk. However, due to the lack of a definitive polysomnographic diagnosis, it is not possible to calculate the sensitivity, specificity or positive/negative predictive values. Further study is required to determine what proportion of patients identified as being at high risk has clinically significant sleep apnea. 
Patients in high vs low risk group as identified by Berlin Questionnaire

\begin{tabular}{|c|c|c|c|}
\hline & $\begin{array}{l}\text { Sample } \\
\text { Overall }\end{array}$ & $\begin{array}{l}\text { Berlin } \\
\text { High Risk } \\
\text { Group }\end{array}$ & $\begin{array}{l}\text { Berlin } \\
\text { Low Risk } \\
\text { Group }\end{array}$ \\
\hline $\begin{array}{l}\text { Number of } \\
\text { patients }(\%)\end{array}$ & 318 & $76(23.9)$ & $242(76.1)$ \\
\hline Sex (\% male $)$ & 53.5 & 56.6 & 52.5 \\
\hline *Mean in age (sd) & $54(16)$ & $58(14)$ & $53(16)$ \\
\hline *Mean in BMI (sd) & $28(5)$ & $32(4)$ & $26(4)$ \\
\hline $\begin{array}{l}\text { *Hypertension } \mathrm{n}(\%) \\
\text { (overt or treated) }\end{array}$ & $116(36.5)$ & $49(64.5)$ & $67(27.7)$ \\
\hline $\begin{array}{l}\text { Prior diagnosis of } \\
\text { sleep apnea } \mathrm{n}(\%)\end{array}$ & $10(3.1)$ & $10(13.2)$ & $0(0)$ \\
\hline
\end{tabular}

